Meta

Journal des traducteurs

Translators' Journal

\title{
Mathematics, Language and Translation
}

\section{Sundar Sarukkai}

Volume 46, numéro 4, décembre 2001

URI : https://id.erudit.org/iderudit/004032ar

DOI : https://doi.org/10.7202/004032ar

Aller au sommaire du numéro

Éditeur(s)

Les Presses de l'Université de Montréal

ISSN

0026-0452 (imprimé)

1492-1421 (numérique)

Découvrir la revue

Citer cet article

Sarukkai, S. (2001). Mathematics, Language and Translation. Meta, 46(4), 664-674. https://doi.org/10.7202/004032ar

\section{Résumé de l'article}

La langue mathématique utilise la langue naturelle. Les symboles se rapportent eux aussi aux termes de la langue naturelle. Les textes mathématiques relèvent de combinaisons de symboles, de langue naturelle, de représentations graphiques, etc. Pour arriver à une lecture cohérente de ces textes, il faut passer par la traduction. Les mathématiques appliquées, comme la physique, passent continuellement d'une langue (et culture) à une autre, et par conséquent, elles sont mieux comprises quand elles relèvent du domaine de la traduction.
Ce document est protégé par la loi sur le droit d'auteur. L'utilisation des services d’Érudit (y compris la reproduction) est assujettie à sa politique d'utilisation que vous pouvez consulter en ligne.

https://apropos.erudit.org/fr/usagers/politique-dutilisation/ 


\title{
Mathematics, Language and Translation
}

\author{
SUNDAR SARUKKAI \\ Indian Institute of Science Campus, Bangalore, India
}

\begin{abstract}
RÉSUMÉ
La langue mathématique utilise la langue naturelle. Les symboles se rapportent eux aussi aux termes de la langue naturelle. Les textes mathématiques relèvent de combinaisons de symboles, de langue naturelle, de représentations graphiques, etc. Pour arriver à une lecture cohérente de ces textes, il faut passer par la traduction. Les mathématiques appliquées, comme la physique, passent continuellement d'une langue (et culture) à une autre, et par conséquent, elles sont mieux comprises quand elles relèvent du domaine de la traduction.
\end{abstract}

\begin{abstract}
The mathematical discourse is not possible without a fertile use of natural language. Its symbols, first and foremost, refer to natural language terms. Its texts are a combination of symbols, natural language, diagrams and so on. To coherently read these texts is to be involved in the activity of translation. Applied mathematics, as in physics, constantly shifts from one language (and culture) to another and, therefore, is best understood within the ambit of translation studies.

\section{MOTS-CLÉS/KEYWORDS}

mathematical discourse, natural language, symbols, translation studies
\end{abstract}

Mathematics is a unique discourse, as represented by its objects of discourse, discursive strategies and even in the way it 'uses' language. But there should be no doubt that the uniqueness of the discourse is not only due to a privileged symbolic system. Of course, mathematics uses symbols as part of its discourse and creates a rich narrative based on these symbols and operators. The central role of the $=$ sign is also an essential difference between this and other discourses. Because of the overwhelming presence of symbols, the specialty of mathematics has been mistakenly attributed to these 'non-linguistic' symbols. This has led to discourses attempting to mimic the mathematical one primarily by appropriating the suspicion of language and attempting to rewrite the discourse in symbolic notation. At the outset, it can be stated that mathematization does not have to do only with appropriating mathematical equations and techniques. It is the engagement with language manifested in its symbolic domain that suggests the uniqueness of the mathematical discourse. From this it follows that one can conceivably have mathematized discourses without it being visibly symbolic.

The emphasis on the symbolic content of mathematics also has a concomitant rejection of natural language $(\mathrm{NL})$. We need to pursue this point further. What is the relationship, at the level of discourse and texts, between NL and symbolic 'language'? Is there 'really' an absence of NL in the texts of mathematics? If NL is present then what role does it play? Wittgenstein addresses the link between NL and mathematics in some detail. For example, Shanker (1989: chap 5) isolates some of these comments 
while pointing out that Wittgenstein's comments on the relationship between 'prose' and mathematics were largely concerned with the role of philosophy of mathematics. For Wittgenstein, the problem of prose in the context of mathematics arises when we talk 'about' calculation. The presence of words makes visible the ever-present problem of conceptualization. Shanker (Ibid., 206) also suggests that Wittgenstein acknowledges that "prose translations of calculations" are translations "in which proofs are presented in a different medium which might 'cast some light on' (i.e., suggest a new way of looking at) the importance or implications of a proof." The basic point of contention is whether mathematics could be done without any recourse to words or whether the prose content is dispensable in doing symbolic mathematics. Shanker (Ibid., 207) is categorical in stating that "it is quite obvious that the presence of 'prose' in the body of proofs is neither arbitrary nor dispensable." I cannot see any point of argument with this position.

Anybody who opens a text of mathematics will note that there is a large percentage of NL (I will stick to English from now on) terms in the text. This is partly, and perhaps ironically, because the erasure of calculations (except for its traces) in the text reduces the symbolic density of the text. But the English subtext of the mathematical text continues to be prominent. Words, phrases and other expressions belonging to the English language play a significant role in the articulation of the mathematical discourse. Let me consider a few examples, from texts that are of advanced level and addressed also to the professional mathematician. I point this out in order to emphasize that the English sub-texts are important in a professional text and not just in semiprofessional or even pedagogical ones. Let us first consider one of the most important theorems in arithmetic called the Fundamental theorem of arithmetic (Apostol, 1976:17):

Fundamental theorem of arithmetic. Every integer $\mathrm{n}>1$ can be represented as a product of prime factors in only one way, apart from the order of factors.

Obviously this theorem is a statement 'belonging to English' in the sense that its meaning is derived from that of the English words used in the statement. There are also terms 'belonging' to mathematics which are written 'in' English. These include 'integer,' 'prime,' 'factors' and 'product.' These are all words that occur in our normal English language use but in this theorem they have a specific meaning. Prime refers to prime numbers which are distinguished by a special property (that of being divisible only by themselves and 1). The word 'factor' refers to the property of expressing a number as a product of other numbers and 'product' itself refers to multiplication. This is a simple example of an important theorem in arithmetic that is expressed in English and depends on it for its articulation. But even as it does this there are also constraints placed on the meaning of certain words. Now this theorem itself is preceded by definitions where each of these terms, like prime, are defined. The statement of the theorem is not symbolic but works by restricting the semantic possibilities of some words used. In this sense, it is in 'essence' symbolic but is not expressed in that manner. Note that the other words also play an important role in generating meaning for this theorem. The phrase 'can be represented' is not defined in the same exact way that words like prime and factor have been. Thus our reading of these terms is open to interpretative possibilities as they occur in English. This is a typical structure of a mathematical statement. When English words are used, some 
of them are strictly bounded in terms of what they refer to or mean. But there is also a surplus of other words that occur as they do in many other discourses based entirely on English. Thus as much as meaning is sharpened at the site of some words the plurality of meanings at others is left as they are. This leads to a very interesting juxtaposition of words.

We should note here that although there is a restriction in the meaning of the words prime, factor etc., there is simultaneously the development of a narrative about prime numbers that is in itself semantically rich. This suggests that it is not 'semantic ascent' that we need to consider, but 'semantic diffusion,' where there is a transfer of meaning 'horizontally' from one domain to another.

Theorems and results following from the above theorem show more symbolic content in the text. But in each case the reduction to a greater symbolic content is first of all made possible by the definitions and the results of preceding theorems. These definitions and the primary results use English much more than the ones that follow and refer to them. So when we see a theorem which is symbolically loaded, each of these symbolic terms has derived its meaning only through the link with English. That is, it is English that speaks mathematics first, prior to the voice of the symbols.

Mathematicians may not take kindly to this view, since it has been a strongly held belief in that community that words are redundant and can always be replaced by symbols. Hadamard's (1996: 75) observations on the psychology of mathematics are pertinent in this context. After mentioning that he fully agrees with Schopenhauer's claim that "thoughts die the moment they are embodied by words," he goes on to say that he believes this of not only words "but even about algebraic signs"! He goes on to add that in the creative act he himself works not in terms of words but vague images in his mind and that it is only in the 'verifying' stage that he "may use algebraic symbols" (Ibid., 82). He continues, "As to words, they remain absolutely absent from my mind until I come to the moment of communicating the results in written or oral form" (Ibid., 82). He cites more examples of mathematicians who hold similar beliefs about the role of words but, as an exception, he quotes another wellknown mathematician, G.Polya who writes to him (Ibid., 84-85):

I believe ... that the decisive idea which brings the solution of a problem is rather often connected with a well-turned word or sentence. The word or the sentence enlightens the situation, gives things, as you say, a physiognomy. It can precede by little the decisive idea or follow on it immediately; perhaps, it arises at the same time as the decisive idea....The right word, the subtly appropriate word, helps us to recall the mathematical idea, perhaps less completely and less objectively than a diagram or a mathematical notation, but in an analogous way...It may contribute to fix it in the mind. (my emphasis.)

Thus although Polya acknowledges the role of language one can see that he is very qualified and hesitant about his acknowledgement (note the use of phrases which are italicized). It is also somewhat ambiguous because although he says that the 'decisive idea' is 'connected' with a word or sentence, he qualifies it by adding that it should be 'well-turned.' Although Polya is not talking about the explicit writing of the discourse but rather about the creative process of doing mathematics, his remarks are applicable for the written discourse as much as to the creative process itself. 
Let us consider another example. Apostol (1976: 129), while describing groups writes about one property of the groups in the following manner: "For every a and b in $\mathrm{G}$, a.b is also in G." We can rewrite this symbolically as $\forall a, b \in G$, a.beG. Has this symbolic rewriting removed the content of English? No, because the symbols are defined prior to their use. So before we are able to write it in the symbolic form we would say, Let $\forall$ stand 'for all' and $\in$ stand for 'belongs to.' Obviously there is some laxity here. In this symbolic notation 'belongs to' and 'in' are used synonymously. We can refine this further by generating various symbols, but the point is that symbols in mathematics first and foremost refer to words in natural language. We should also note the shifting meanings arising from the use of this 'translation.' Thus $\in$ stands for 'belongs to,' 'belonging to' and so on. If we re-translate the symbols back into English, they would read: For every a, b belonging to $\mathrm{G}$, a.b (also) belongs to $\mathrm{G}$. This kind of dynamic interchange between the English sub-text and the symbolic one causes a constant slippage of meaning and thus opens up the semantic universe of this discourse.

The above discussion suggests that the primary reference of the mathematical discourse is to NL. This referred-to 'sub-language of English' is not a discourse by itself but is largely a collection of words and phrases in that language. But they play an important role, if not in the calculations per se, then at least in the opening up of semantic possibilities to the creative imagination. ${ }^{1}$ This also brings mathematics into connection with the real life-world from which it arises in the first place.

If we go through any mathematical text we find some key terms and phrases which occur repeatedly. The common examples are Let, If, Assume, Suppose, Easily verified that, Follows that, Can see, and so on. The mathematical discourse also has a large number of 'names' that are identified and associated with some special properties of numbers or functions. English is also put to rhetorical use. Terms like 'easily verified that,' 'can see' and so on reinforce the presence of rhetoric in the writing. It seems as if the English language is used as a 'carrier' for these rhetorical moves. It also helps to introduce spaces of ambiguity where explicit metaphorical moves can be made. When this happens new results are produced which are not 'strictly' mathematically correct but then they get taken up and refined to fit into the mathematical scheme. There are numerous such examples where mathematics has refined and made more concise terms in physics that were mathematically unsound.

The presence of English in mathematics occurs across disciplines. Consider this example from topology. "A homeomorphism, or topological transformation, is a continuous one-to-one mapping of a topological space $\mathrm{X}$ onto a topological space $\mathrm{Y}$ such that $\mathrm{f}^{-1}$ is also continuous" (Kelley, 1955: 87). This statement is a definition of 'homeomorphism.' The words in English here function 'literally' like names. As names, they are not the sites of plurality but are 'fixed.' In the usual case of names, the 'fixed' nature of names is due to a reference to a thing that is so named. Here there is a simulation of names, in that these 'names' are not available for participation in the language in which they are written (English). But it cannot be written, communicated and taken over into the discourse of mathematics without the presence of the written script of English. This may perhaps suggest that English words are themselves used as graphic symbols devoid totally of meanings other than those derived through 'naming.' But this too cannot be correct. For look at the use of words like 'continuous,' a central term in analysis. Although there is a particular meaning to 
'continuous' it cannot be replaced by any other empty-signifying word because mathematics wants to hold onto the semantic image inspired by the word 'continuous' as it occurs in non-mathematical talk. This holding onto the semantic domain of English but rewriting the discourse in a way that simulates rejection of plurality through 'precise' definitions is a defining mark of the mathematical discourse. It constitutes the simulation of restricting the spread of meaning even while surreptitiously encouraging it! This is the mediation, the very act of translation, which is bi-valent.

The distillation of meaning from words already places the words on par with symbols. So the English words here mimic the script of the language but they do not strictly 'belong' to the culture of that language. The writing of this discourse attempts to erase the language but keeps the traces of this erasure. But having done this it also allows the word, which has been reduced to a 'single' meaning, to be taken up in a 'play of signifiers' in a different discourse with a different set of rules.

This then is the contention: symbols as used in mathematics first come into being only with respect to and with the help of words in NL. These symbols first refer to the words before anything else. The process of symbolization does not add anything more to the meaning of the word with which the symbol is associated; in fact by the process of strict definitions, it pares down the multiple meaning of words. Then what is it that the symbolization does which words cannot? The symbolization allows operations to be performed on them and in this process of manipulating, building and doing, they create newer expressions. The operators themselves work under the regulative force of the $=$ sign .

But even in this activity we are never far from falling into the 'dangers' of the expressive richness of NL. If symbolization is seen as an activity attempting to curtail the many meanings that a word inspires, then the complexity of the mathematical discourse stands as a proof that it has failed in its attempts! For in the realm of imagination, in the mind of the mathematician, the presence of NL terms cannot shake away this semantic plurality or the metaphoric force of their utterance. One can always transfer back these images into symbolic ones. The discipline of mathematics does not inhere in its denial of new images. Rather, they are welcome as long as any new entrant conforms to the accepted principles. Thus metaphoric imagination is a must; ambiguity of images is wonderful as long as they are all prior to the symbolic process.

The above discussion on the link between mathematics and NL continuously flirts with the idea of translation, even if this image is not made explicit. The emphasis on the notion of writing, that which makes mathematical discourse possible and unique, naturally leads us to consider the question of translation with reference to mathematics. The link between translation and mathematics, always hidden, needs to be explicated because it informs us that the activity of mathematics is indeed a writing of mathematics and that this writing attempts to erase the notion of translation as much as it remains indebted to it. This is the link between mathematics and translation that needs to be examined.

It is understandable that mathematics would like to distance itself from the 'idea' of translation. Many factors suggest this. Firstly, is the excessive emphasis on truth, forgetting the fact that the pleasure of writing mathematics in terms of calculation has a seduction that goes beyond the legitimacy of the end result. The activity is intensely satisfying and the end result is a bonus. Secondly, the suspicion of language. 
Mathematics carries this suspicion to an extreme where words are literally reduced to linguistic points - into alphabets. Translation creates a double dose of suspicion because its activity inspires little confidence and respect, even among those whose concerns are within the boundaries of NL, largely because it is seen to be derivative and unable to capture the original expression. Mathematics considers NL itself to be notoriginal as far as the Platonic world is concerned. Under this double onslaught, it is no wonder that there is little acknowledgement of the link between mathematics and translation.

But mathematics is writing. The activity by which mathematics creates its alphabets highlights its first engagement with NL: it reduces the graphic width of words into graphemes. This move is a writing of writing. It is rewriting the already written. It is the activity of translation which best describes this 'writing of writing.' First and foremost, translation is the writing of the written and first and foremost, the writing of mathematics is an activity of translation.

The conventional theories of translation have been too absorbed with meaning. Words are seen as marks that stand in place of a larger meaning. In making this move, they forget the originary impulse of words as graphic marks on paper. Before we enter the domain of meaning, we enter the world of perception of the written, the sight of the squiggles on the sheet. Translation at this level is akin to tracing. What do we do when we trace other than to learn to reconstruct the graphic marks? The mark is the original and every attempt to reproduce it is a trace in this sense. Mathematics, beginning first with the reduction of the word-mark into an alphabet-mark, voices this activity clearly: it is tracing which first suggests the possibility of translation.

The symbolic domain is a language, based as it is on conventions and rules. These symbols generate meaning, create texts and discourses. They also create incredibly rich narratives about the symbolic world. But, as discussed earlier, the symbolic world is generated through its reference to the world of NL. NL is involved in the creation of this language, yet is found to be totally unsuitable as a partner in its discourse. So NL has to be kept invisible, yet the writing of its text betrays it and makes NL visible, overly visible. Then the recourse is to negate any 'substantial' content to it. Mathematics gives into a metaphysical duality: it is the symbolic world which is the mind of mathematics and the written world, both the terms of NL and the hidden texts of calculation, the 'body' of the discourse. In such a scenario, how can the possibility of translation come up? In invoking the name of translation, we are invoking and creating the image of $\mathrm{NL}$ as the original, and the symbolic language not merely as an abridged form of it but as a translated form of it. Can anything be more blasphemous? But can anything be truer?

How to further question this issue? NL cannot be accepted (by those who see mathematics entirely in terms of logic and Platonism) as the original out of which the text of mathematics arises through some kind of translation, not least because mathematics presumably refers to a Platonic world. Unlike other discourses, not only does mathematics negate any links with NL, it also launches a devastating critique of the ambiguity and confusion inherent in NL. So all traces of its link need to be erased. But it can do so only through the act of writing, and it is this writing which recaptures the link and suggests that the language of mathematics is itself a translation of NL.

More than other languages in which translation is involved, it is the link between NL and mathematics, exhibited both in 'pure' and 'applied' mathematics, which 
comes closest to Walter Benjamin's thematization of translation in terms of kinship of language and pure language. ${ }^{2}$ It is the kinship of languages that makes translation possible and in fact can be seen to be initiatory of translation. The kinship of languages is not the kinship derived from common origins, or common scripts or membership to families. The possibility of translation itself is to be found in the "central reciprocal relationship between languages" (Johnston, 1992: 44). The idea of kinship is one that is naturally inspired by this interplay between mathematics and NL. Kinship of languages, for Benjamin, is not concerned with the notion of similarity between them, but more importantly with "the intention underlying each language as a whole-an intention, however, which no single language can attain by itself but which is realized only by the totality of their intentions supplementing each other" (Ibid., 44).

It is quite clear that mathematics as a 'language' is not exhausted by the symbolic content alone but also needs NL to 'supplement' it. In the case of mathematics, the kinship is made 'visible' at the level of the graphical mark itself. It is made more visible in the reference of symbols to words in NL. Associated with the theme of kinship of languages is the idea of 'pure' language, which is undoubtedly a problem in Benjamin's writings, not least because of its association with theological doctrines. ${ }^{3}$ But his incomplete articulation of pure language resonates closely with the many views on mathematical language, held by both scientists and mathematicians. As Johnston (Ibid., 44) remarks, "If there is a language of truth or a true language, Benjamin states, then it is this 'pure language." For Benjamin, the notion of pure language arises in the context of translation; for science and mathematics it arises in the context of its untranslatability! For the proponents of this position, mathematics is a privileged language, one that speaks the truth. Even nature is seen to be written in the language of mathematics. The claims of truth in mathematics, the rigor of proofs and the difficulties of calculation, have all gone towards privileging mathematics as the language of truth. And if we look at what is at stake in the notion of truth, it is the untranslatability of it, while, ironically for Benjamin, the idea of 'pure language' articulates the possibility of translation between different languages. And what is unique about mathematics? It is the inexpressibility of it in any other language; the impossibility of translating it into other languages. Thus, in this context, the defining moment of mathematics, its claim to fame in contrast to other languages is this: mathematics continuously attempts to negate translation. If this were not so, it could always be translatable into other languages, and hence seen to be a natural language itself. But the discourse of mathematics is such that it betrays this attempt to render itself untranslatable. Why else would a discourse write and present itself in such an exclusionary fashion? Why else would the members of the community have to continuously prove their worth not only in their creative expression but also in learning to read its texts by writing them simultaneously? Why does mathematics make a virtue out of its exclusivity? Is it just to keep it outside the orbit of translation into other languages?

The writing of mathematics itself does not allow for this claim to go unchallenged. What the discourse reflects is that the two languages (and also the use of diagrams, figures and so on) that constitute mathematics inhabit different cultural spheres. The language and the discursive customs of these two languages are seemingly far apart. But a good translation is one that responds to the movement from 
one culture to another by not 'majoritizing.' ${ }^{\text {Th }}$ The very ability to let symbols speak for words, then get operated, calculated and transformed and then again called upon to speak for other words, shows the inherence of translation in the creation of the mathematical discourse. Here I find Paul de Man's comment on Benjamin's essay incisive and particularly illuminating in understanding the 'essential tension' which pervades mathematics in its relationship to natural languages. He writes (1986: 84):

What translation does ... is that it implies ... the suffering of the original language. We think we are at ease in our own language, we feel a coziness, a familiarity, a shelter in the language we call our own, in which we think that we are not alienated. What the translation reveals is that this alienation is at its strongest in our relation to our own original language, that the original language within which we are engaged is disarticulated in a way which imposes upon us a particular alienation, a particular suffering.

The writing of mathematics shows that as much as words are sites of differential plurality so too are symbolic reductions of them. But the plurality exhibited in this symbolic reduction belongs to a different domain altogether. Numbers (as words) cannot be multiplied. But numbers as 9 or $\mathrm{n}$ can be. Energy (as word) cannot be differentiated but energy as E can be. Velocity (as word) cannot be added component wise, but velocities as vectors can be. And so on and on. In the case of using mathematics to voice the world, it becomes a mixture of the world, language and mathematics. The continued translation from one to the other is as much the source of creativity as it is of confusion.

There are two further remarks on this 'kinship' between mathematics and translation. One is the need to understand 'applied' mathematics (as used in physics and increasingly in other disciplines) through the imagery of translation. The other involves introspection on the equality sign in the context of translation. Before I discuss the former, let me make a few passing remarks on the relation of the equality sign and translation. It is well known that the notion of translation has a necessary association with the ideas of comparison. What makes translation studies so difficult is not only the complexities of language and the indecidability of texts but the difficulty in getting a handle on what comparison between texts and texts, and texts and language could mean. There are two specific cases of comparison embodied in the mathematical discourse. One is the notion of tracing alluded to before. The other is the presence of the $=$ sign. $=$ is primarily an agent of comparison. Between numbers, between functions ... between texts? The = sign has its own dynamics and metaphysical baggage. ${ }^{5}$ It regulates the discourse even as it frees its imagination. It speaks to mathematics as much as it speaks to translation. It is the exemplar of comparison and as such shows the complexity and pitfalls of comparison itself.

There is also a need to understand 'applied' mathematics through the imagery of translation for the following reasons. Perhaps the strongest support for the claim that mathematics is not only a unique 'language' but also one with a special connection to 'truth' comes from the position best articulated as the indispensability thesis.' Linked with Putnam and Quine, this thesis asserts that the existence of mathematical entities is strongly suggested by their indispensability in science, through their indispensable role in the scientific explanations of the world (Field, 1989). This statement is essentially one that asserts the existence status of these entities, primarily as Platonic ones. But there is a larger issue involved here that is at the level of language. This 
thesis suggests that, in the final analysis, the belief in mathematics as the 'language of truth' is largely dependent on the 'success' of science. The success of science lies in its claim to describe the world 'as is' and the concomitant exhibition of this description through technological intervention in the world. This is then taken to suggest that mathematical statements speak 'truth,' not merely about Platonic entities, but also about the physical world. This claim is not merely about the existence of mathematical entities, but also a statement about the truth content of its expressions. Once we realize that the indispensability thesis has as much to do with epistemological claims as ontological ones, then the shift to language is immediate. In particular, the notion of 'applied' strongly implies a movement of languages inhabiting different cultural domains, with their respective conventions, rules and meaning. Thus to explain the use of mathematics in scientific narratives is to first acknowledge the activity of translation which makes possible this 'motion' across linguistic cultures. We have to see translation under the broader rubric of culture: "Since languages express cultures, translators should be bicultural, not bilingual" (Lefevere and Bassnett, 1990).

It is easy to see why applied mathematics is an activity of translation. The cultures of physics and chemistry, for example, are very different from the symbolic and calculational culture of mathematics. Their languages, methodologies, narratives and discourses are all different. The cultural difference between the experimentalists and theorists, as described by Galison (1997), for example, is a difference manifested in the different languages (along with the different practices) used by these communities. In the case of mathematics and physics, for example, the gap is perhaps wider. The physicists' view of the mathematical discourse is quite different from the mathematicians' view. Physicists are usually not concerned with the existence theorems, the intricate details of a proof of some result and so on. Largely, they find in the mathematical discourse results that they appropriate in the construction of mathematical models of the physical system they are modeling. Now this appropriation is not just a 'taking over' but rather involves creative 'rewriting' into the language of physics. This activity is an activity of translation and the problems in it are similar to the problems arising in translating from one language to another. Duhem states this explicitly (1974: 133):

Thus as both its starting and terminal points, the mathematical derivation of a physical theory cannot be wedded to observable facts except by a translation. In order to introduce the circumstances of an experiment into the calculations, we must make a version which replaces the language of concrete observation by the language of numbers; in order to verify the results that a theory predicts for that experiment, a translation exercise must transform a numerical value into a reading formulated in experimental language. But translation is treacherous. Between the concrete facts, as the physicist observes them, and the numerical symbols by which these facts are represented in the calculations of its theorists, there is an extremely great difference.

Many physicists, in their use of mathematics, attempt to distill the essence of mathematical results without worrying about the intricate mathematical details. Not only is the essence distilled in the form of particular results and proofs, but it is also then translated back into the language of the physical system being modeled. This move of translation allows for new and fertile narratives to develop. We should remember here that "translation, like all (re)writings is never innocent. There is always a context 
in which the translation takes place, always a history from which a text emerges and into which a text is transposed" (Lefevere and Bassnett, 1990: 11).

Consider the example of symmetry. Groups are the mathematical structures associated with the idea of symmetry in physics. Groups, as mathematical structures, are historically prior and were available for the articulation of the modern concepts of symmetry. But the way in which these structures are written and used differs quite markedly in the discourses of mathematics and physics. In the case of symmetry, group theory has been translated into the language of physics. In doing this, it is believed that essential ideas are retained. This belief is possible only because we have historically held onto such beliefs in translation. And now, with newer interpretations of translation, we are made aware that it is quite impossible to isolate essences that can then be translated. This suggests two points: firstly, mathematics is unique because it somehow allows essences to be distilled, isolated and then translated and transmitted. Secondly, if we do not give into this position, it suggests that perhaps group theory is not the only mathematical expression of symmetry and further, that more radical and free translations between mathematics and physics will generate other structures to describe symmetry. The vindication of the latter will have to come from physics itself, a physics which understands its activity through the alternative prism of translation.

It is perhaps apt that I end this discussion with a reference to Walter Benjamin's view on the difference between languages and their connection to translation. For, it is indeed the case that applied mathematics lives at the edge of two languages, yet inhabits both the languages in full. One is the language of the symbolic world called mathematics and the other is the language of the world, of the human and the nonhuman alike, used to express, communicate and discourse. These languages are no doubt different but the difference is to be welcomed. The last words should be Benjamin's:

The difference between languages that translation must somehow necessarily overcome cannot and should not be suppressed, for translation lives on (or in) this difference; a particular translation will be valuable according to how it alludes to or dissimulates this difference, or more positively, reveals and accentuates it (Johnston, 1992: 46).

\section{NOTES}

This work was supported by the Homi Bhabha Fellowship and is part of a larger work on science, language and translation. I am indebted to Michael Weinstein for a careful reading of the manuscript and for his many suggestions. This material will appear in my book titled "Translating the World: Science and Language" published by University Press of America, Lanham, to appear, 2002.

1. To quote Shanker (1989: 209): "Wittgenstein readily agreed that ... verbal concepts can and do play an integral role in the development of mathematical ideas."

2. See Walter Benjamin (1992) and also Andrew Benjamin (1989).

3. In this context, see Johnston (1992), de Man (1986), and Derrida (1985).

4. See Venuti (1998).

5. I have discussed this in more detail in an unpublished paper of mine titled "The Writing of Mathematics." 


\section{REFERENCES}

Apostol (1976): Introduction to Analytic Number Theory. Springer-Verlag.

Benjamin, A. (1989): Translation and the Nature of Philosophy: A New Theory of Words. London and New York: Routledge.

Benjamin, W. (1992): “The Task of the Translator," Illuminations. Trans. Harry Zohn, Fontana Press.

De Man, P. (1986): The Resistance to Theory. Manchester: Manchester University Press.

Derrida, J. (1985): “Tours de Babel," Difference in Translation, Joseph Graham (ed.), Ithaca: Cornell University Press.

Dunem, P. (1974): The Aim and Structure of Physical Theory. New York: Atheneum.

Field, H. (1989): Realism, Mathematics and Modality. Oxford: Blackwell.

Galison, P. (1997): Image and Logic. Chicago and London: University of Chicago.

Hadamard, J. (1996): The Mathematician's Mind. Princeton: Princeton University Press.

Johnston, J. (1992): “Translation as Simulacrum," Rethinking Translation, Venuti (ed.), London and New York: Routledge.

Kelley (1955): General Topology. Van Nostrand Company.

Lefevere, A and S. Bassnett (1990): "Proust's Grandmother and the Thousand and One Nights," Translation, History and Culture, Bassnett and Lefevere (eds.), London and New York: Pinter Publishers.

Shanker, S.G. (1987): Wittgenstein and the Turning-Point in the Philosophy of Mathematics. London and Sydney: Croom Helm.

Venuti, L. (1998): The Scandals of Translation. London and New York: Routledge. 